\title{
Anti-inflammatory Potential of a Novel Imidazole Containing Murrayanine Based Chalcone
}

\author{
Debarshi Kar Mahapatra ${ }^{1 *}$ and Ruchi S Shivhare ${ }^{2}$ \\ ${ }^{1}$ Department of Pharmaceutical Chemistry, Dadasaheb Balpande College of Pharmacy, India \\ ${ }^{2}$ Department of Pharmaceutical Chemistry, Kamla Nehru College of Pharmacy, India
}

*Corresponding author: Debarshi Kar Mahapatra, Assistant Professor, Department of Pharmaceutical Chemistry, Dadasaheb Balpande College of Pharmacy, Nagpur 440037, Maharashtra, India

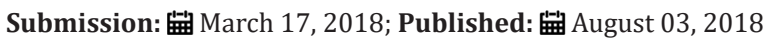

\begin{abstract}
Murrayanine is an active carbazole compound present in the Indian Curry tree, known as Murraya koenigii L. belonging to the family Rutaceae. Traditionally, it is known to exhibit anti-anemic, febrifuge, carminative, stomachic, purgative, astringent, and anthelmintic. The present research involved the synthesis of (E)-3-(4-(1H-imidazol-1-yl)phenyl)-1-(1-methoxy-9H-carbazol-3-yl)prop-2-en-1-one from murrayanine and 4-(imidazol-1-yl) acetophenone and exploring the anti-inflammatory activity of the fabricated hybrid. The current effort involved screening the significance of imidazole function present in the ring-B of the murrayanine-chalcone scaffold and their further optimization thereof with emphasis on structural aspects. A moderate edema reducing potential has been seen in the newly developed compound (3). As compared with the indomethacin, the standard drug, the chalcone exhibited impressive anti-inflammation effect after $3 \mathrm{hrs}$. The activity may be mediated by inhibiting the pro-inflammatory constituents like cyclooxygenase-2 (COX-2) and lipoxygenase (5-LOX) which may be due to the imidazole group and benzylidene acetophenone scaffold. This study will open new avenues of research by promoting the development of more potent natural product based hybrid compounds with a high level of safety. In successive studies, more derivatives may be prepared to rationally study the structural influence over the activity and to derive information which will be of applied interest.
\end{abstract}

Keywords: Murrayanine; Murraya koenigii; Imidazole; Chalcone; Inflammation; Synthesis

Abbreviations: COX-2: Cyclooxygenase-2; LOX: Lipoxygenase; DEC: Department Ethical Committee; TMS: Tetra Methyl Silane

\section{Introduction}

Murrayanine is an active carbazole compound present in the Indian Curry tree, known as Murraya koenigii L. belonging to the family Rutaceae [1]. In general, the extract comprises of murrayanine, euchrestine B, mahaninebine, mahanine, bismahanine, koenimbine, O-methylmahanine, bispyrayafoline, mahaninebicine, O-methylmurrayamine A, isomahanine, bismurrayafoline, etc [2]. Traditionally, it is known to exhibit anti-anemic, febrifuge, carminative, stomachic, purgative, astringent, and anthelmintic [3]. Murrayanine is the extremely investigated molecule due to the simplicity of the chemical characteristics, an application template promoting semi-synthesis, facilitates substitution at multiple sites, and its resultant therapeutic aspects, etc [4]. Mother Nature has all the solution for all the prevailing ailments, and therefore naturebased scaffold/skeleton will definitely lead to the development of potent inhibitor molecules [5].

In our previous research, murrayanine was explored exhaustively for developing several promising semi-synthetic derivatives or hybrid compounds rationally with enhanced pharmacological effect, reduced toxicity, and better target modulator perspective. The hybrids of thiadiazole, oxazole, hydantoin, thiazole, pyrimidine, pyrrole, isoxazole, benzodiazepine, benzothiazepine, benzoxazepine, phthalimide, etc. were fabricated which demonstrated better therapeutic potentials like antibacterial, anti-fungal, anti-oxidant, anti-inflammatory, anti-diabetic, anti-anxiety, anti-convulsant, etc [6-14]. The present research involved the synthesis of (E)-3-(4-(1H-imidazol-1-yl)phenyl)-1(1-methoxy-9H-carbazol-3-yl)prop-2-en-1-one from murrayanine and 4-(imidazol-1-yl)acetophenone and exploring the antiinflammatory activity of the fabricated hybrid. The current effort involved screening the significance of imidazole function present in the ring-B of the murrayanine-chalcone scaffold and their further optimization thereof with emphasis on structural aspects.

\section{Materials and Methods}

\section{Chemicals and instrumentation}

The starting compound, murrayanine was procured by extraction from Murraya koenigii L. by the soxhlation process as previously reported by our group [15]. The reactant 4-(imidazol1-yl) acetophenone was purchased from Sigma-Aldrich, Germany. The analytical grade reagents and solvents used during synthesis 
were purchased from HiMedia Ltd., Mumbai, India. The progress of the reaction was monitored by Merck pre-coated TLC plates of Silica Gel-G. The FT-IR study was performed in Shimadzu ${ }^{\circledR}$ IRAffinity-1 (KBr system). The ${ }^{1} \mathrm{H}-\mathrm{NMR}$ was obtained on Bruker Avance-II instrument employing tetramethylsilane (TMS) as the internal standard. The mass spectra were recorded on MICROMASS Q-TOF instrument. The PerkinElmer 2400 model Elemental Analyzer was utilized for CHN analyses.

\section{Animals}

Same-sex albino rats of 5-6 weeks age, having body weight range of $140-230 \mathrm{~g}$ were taken for the experiment after getting permission from the Department Ethical Committee (DEC) as well as CPCSEA (1389/a/10/CPCSEA). The experimental animals were kept under hygienic conditions in the animal house under controlled environment (temp. 24-25을 $50-60 \%$ RH/and $12 \mathrm{hr}$ light/dark shift). The rats were provided free access to standard rodent feed and water.

\section{Synthesis of target compounds}

The imidazole containing murrayanine chalcone was synthesized from the starting carbazole material, murrayanine (1), extracted from the stem bark of M. koenigii L. The reaction involved reacting the - $\mathrm{CHO}$ portion (aldehyde) of murrayanine with the acetyl part $\left(-\mathrm{COCH}_{3}\right)$ of the imidazole containing acetophenone (2) in the presence of the ethanolic $\mathrm{NaOH}$ solution. The plausible mechanism of chalcone formation was an aldol condensation which includes enolate ion reaction with the carbonyl function to derive a $\beta$-hydroxyketone followed by a dehydration to yield enone component (3). The (Figure 1) depicts the reaction for the synthesis of chalcone derivative.<smiles>COc1cc(C=O)cc2c1[nH]c1ccccc12</smiles>

1
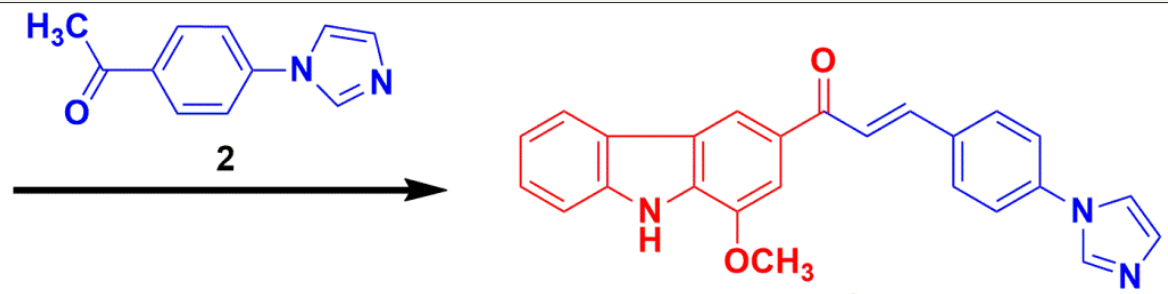

3

Figure 1: Synthesis outline of novel imidazole containing murrayanine based chalcone.

Synthetic protocol for (E)-3-(4-(1H-imidazol-1-yl)phenyl)-1-(1-methoxy-9H-carbazol-3-yl)prop-2-en-1-one (3)

Murrayanine (1) and 4-(imidazol-1-yl)acetophenone (2) were made to react in equimolar concentration $(0.01 \mathrm{M})$ in presence of an aqueous solution of sodium hydroxide $(20 \mathrm{~mL})$ containing $90 \%$ ethanol (25mL). The content was made to reflux for the duration of $3 \mathrm{hr}$ and the reaction mixture was kept for the whole night. Following that, the content was poured over crushed ice and the content was separated in presence of dil. $\mathrm{HCl}$ (few drops). The precipitated solid product was washed thoroughly with water, filtered under vacuum using a Buchner funnel, and re-crystallized properly.

73\% yield; FTIR(KBr)U $\left(\mathrm{cm}^{-1}\right): 3217(-\mathrm{NH}$, stretching), 3174(C$\mathrm{H}$, aromatic), $1701(\mathrm{C}=0), 1649(\mathrm{C}=\mathrm{N}), 1663(\mathrm{C}=\mathrm{C}$, aromatic), 1568($\mathrm{NH}$, bending), $1281(\mathrm{C}-\mathrm{N}), 1252(\mathrm{C}-0) ;{ }^{1} \mathrm{H} \operatorname{NMR}\left(\delta, \mathrm{ppm}, \mathrm{CDCl}_{3}\right)$ : 10.28(9, 1H), 7.1-8.6(Aromatic, 13H), 3.89(1, 3H). MS: $\mathrm{M}^{+} 393$. Anal. Calcd. for $\mathrm{C}_{25} \mathrm{H}_{19} \mathrm{~N}_{3} \mathrm{O}_{2}$ : C, 76.32; $\mathrm{H}, 4.87 ; \mathrm{N}, 10.68$. Found: C, 76.17; H, 4.39; N, 10.23 .

\section{Acute toxicity studies}

According to the OECD guideline, the in vivo safety of the molecule in therapeutics/testing is an essential criterion. The acute toxicity study was performed to determine the highest therapeutic dose which will demonstrate no considerable mortality. The study involved progressively increasing doses of the experimental molecule from 25 to $500 \mathrm{mg} / \mathrm{kg}$. The therapeutic dose was decided based on $\mathrm{LD}_{50}$ value [16].

\section{Anti-inflammatory screening}

The in vivo anti-inflammatory activity of the fabricated molecule was studied by carrageenan-induced paw edema method. The experimental protocol was performed in overnight fasted rats to reduce any inconsistency of edema. The rats in the control group received the saline solution containing a few drops of solubilizer (Tween 80). Before initiation of protocol, $5 \mathrm{~mL}$ of distilled water was administered by oral route, individually. The inflammation was created by introducing $1 \%$ carrageenan solution at the right hind paw via the subcutaneous route. The edema reducing potential was screened at a dose of $100 \mathrm{mg} / \mathrm{kg}$ b.w. The compound was initially suspended in saline solution and administered $1 \mathrm{hr}$ previous to the commencement of the study. The edema was measured using mercury digital micrometer for $3 \mathrm{hrs}$ duration with an interval of $1 \mathrm{hr}$. The difference in the dimension of the width of injected and non-injected paws was computed to determine the edema reducing the ability of the experimental compound. The acquired results were expressed as the Mean \pm SEM [17].

\section{Statistical treatment}

The experimentally generated data were analyzed by one-way ANOVA method followed by treatment with Dunnett's multiple comparison test. The value of $\mathrm{P}<0.01$ was considered to be statistically significant. 


\section{Results and Discussion}

\section{Chemistry}

The spectroscopy and elemental analysis studies supported the formation of the compound (3). The disappearance of (-CHO) component and the appearance of $\mathrm{C}=0$ at $1701 \mathrm{~cm}^{-1}$ confirmed the formation of the chalcone. The $\mathrm{C}=\mathrm{N}$ component at $1649 \mathrm{~cm}^{-1}$ substantiates the presence of five-membered imidazole portion. The key FT-IR spectral data have been highlighted in the experimental section. The ${ }^{1} \mathrm{H}-\mathrm{NMR}$ spectra represented few key aspects. The spectral range of 7.1-8.4ppm emphasizes the presence of protons in the compound. Additionally, the $-\mathrm{NH}$ and $-\mathrm{OCH}_{3}$ aspects were located at $10.28 \mathrm{ppm}$ and $3.89 \mathrm{ppm}$, respectively. Furthermore, the mass spectra presented exactly the same molecular weight of the fabricated molecule in the base peak. In addition to that, the fragment peaks (m/z 100-200) also appeared. Finally, the observed ratio(s) of the elements $(\mathrm{C}, \mathrm{H}$, and $\mathrm{N}$ ) of the produced derivative was found to be of immense support.

\section{Acute toxicity study results}

The compound (3) did not show any sign of toxicity with the escalated dose and was seen as a safe compound. The antiinflammatory was screened at a dose of $100 \mathrm{mg} / \mathrm{kg}$ b.w.

\section{Anti-inflammatory screening}

Table 1: In vivo anti-inflammatory potential of $(E)-3-(4-$ (1H-imidazol-1-yl)phenyl)-1-(1-methoxy-9H-carbazol-3-yl) prop-2-en-1-one.

\begin{tabular}{|c|c|c|c|}
\hline Compound & \multicolumn{3}{|c|}{ Percentage (\%) Inhibition of Edema } \\
\hline & $\mathbf{1 h r}$ & $\mathbf{2 h r}$ & $\mathbf{3 h r}$ \\
\hline 3 & $20.54^{* *} \pm 2.19$ & $29.24^{*} \pm 2.32$ & $40.13^{*} \pm 2.77$ \\
\hline \multirow{2}{*}{ Indomethacin } & $46.17^{* *} \pm 1.42$ & $59.66^{*} \pm 1.81$ & $73.91^{* *} \pm 1.34$ \\
\hline
\end{tabular}

$\mathrm{n}=6 ; \mathrm{ED}_{50}$ of $100 \mathrm{mg} / \mathrm{kg}$ bw in male adult albino mice; ${ }^{*} \mathrm{P}<0.01 ;{ }^{*} \mathrm{P}<0.05$

A moderate edema reducing potential has been seen in the newly developed compound (3). As compared with the indomethacin, the standard drug, the chalcone exhibited impressive antiinflammation effect after 3hrs. The activity may be mediated by inhibiting the pro-inflammatory constituents like cyclooxygenase-2 (COX-2) and lipoxygenase (LOX) which may be due to the imidazole group and benzylideneacetophenone scaffold [18]. However, it may be predicted that rationally substituting the murrayanine portion (Ring-A) and the imidazole part (Ring-B) by electron-withdrawing/ donating group may lead to enhanced pharmacological activity, which was awfully missing in this study. The substitution by different moieties comprising of active oxygen and nitrogen atoms may facilitate the binding with the active site of the inflammation mediating targets [19]. Moreover, it may be envisaged that due to the lack of lipophilic groups, the compound (3) did not penetrate or cross the biological membrane to mediate the effect. Therefore, the lipophilic group may play an imperative role in promoting the anti-inflammatory activity (Table 1 ).

\section{Conclusion}

The research presented a semi-synthetic approach for the fabricating of imidazole containing chalcone molecule which demonstrated noteworthy anti-inflammatory activity as compared with the standard drug, indomethacin. However, it may be stated that substitution by electron-withdrawing/donating group at both the rings may facilitate better edema reducing activity due to the oxygen and nitrogen atoms which will promote strong binding with the inflammation mediating targets like COX-2 and LOX. This study will open new avenues of research by promoting the development of more potent natural product based hybrid compounds with a high level of safety. In successive studies, more derivatives may be prepared to rationally study the structural influence over the activity and to derive information which will be of applied interest.

\section{Acknowledgement}

Authors are highly thankful to Savitribai Phule Pune University, Pune, Maharashtra, India for providing research grants (Grant No. 13PHM000126).

\section{References}

1. Chauhan B, Dedania J, Mashru RC (2017) Review on Murraya Koenigii: Versatile role in management of human health. World J Pharm Pharm Sci 6(3): 476-493.

2. Gahlawat DK, Jakhar S, Dahiya P (2014) Murraya koenigii (L.) Spreng: An ethnobotanical, phytochemical and pharmacological review. J Pharmacog Phytochem 3(3): 109-119.

3. Shivhare RS, Mahapatra DK, Nair RR, Deshmukh SN (2016) Schiff's base derivatives of murrayanine demonstrated enhanced anti-oxidant activity than its parent moiety. Indian J Pharm Edu Res 50(3): 9-15.

4. Mahapatra DK, Shivhare RS, Asati V (2018) Locomotor inhibitory activity of some Murrayanine-Chalcone based 2,3-dihydrobenzo[b][1,4] thiazepine derivatives: Exploring Anxiolytic potentials. Chronicle Pharm Sci 2(1): 462-468.

5. Mahapatra DK, Bharti SK, Asati V (2015) Anti-cancer chalcones: Structural and molecular target perspectives. Eur J Med Chem 98: 69114.

6. Mahapatra DK, Das D, Shivhare RS (2017) Substituted thiazole linked murrayanine-Schiff's base derivatives as potential anti-breast cancer candidates: Future EGFR Kinase inhibitors. In J Pharm Sci Drug Res 9(3): 139-144.

7. Mahapatra DK, Shivhare RS, Kumar P (2018) Murrayanine-chalcone transformed into novel pyrimidine compounds demonstrated promising anti-inflammatory activity. Asian J Pharm Res 8(1): 6-10.

8. Mahapatra DK, Shivhare RS, Joseph TM (2017) Design and characterization of Murrayanine linked Isoxazole derivatives: Novel class of bacteriocidal agents. Int J Res Drugs Pharm Sci 1(1): 11-15.

9. Mahapatra DK, Shivhare RS, Bharti SK (2017) Novel Murrayanine based Pyrazole analogs as emerging anti-fungal candidates: Design, synthesis, characterization, and in vitro evaluation. Res Pharm 1(1): 1-5.

10. Mahapatra DK, Shivhare RS (2017) Synthesizing an anti-oxidant principle 2-(((1-methoxy-9H-carbazol-3-yl)methylene)amino)isoindoline-1,3dione from $\mathrm{N}$-aminophthalimide and murrayanine. Inventi Med Chem (4): 1-3. 
11. Mahapatra DK, Das D, Shivhare RS, Borkar SS (2018) Murrayaninehydantoin and -thiohydantoin analogs as promising anti-convulsant agents: Synthesis, Characterization and Molecular Docking Studies. MOJ Bioorg Org Chem 2(2): 47-51.

12. Mahapatra DK, Shivhare RS, Haldar AGM (2017) Novel Schiff's base containing Murrayanine-1,3,4-Thiadiazole Hybrids as potential antiinflammatory agents. Asian J Chem Pharm Sci 2(2): 10-15.

13. Mahapatra DK, Shivhare RS, Gupta SD (2018) Anxiolytic activity of some 2,3-dihydrobenzo[b][1,4] oxazepine derivatives synthesized from Murrayanine-Chalcone. Asian J Res Pharm Sci 8(1): 25-29.

14. Mahapatra DK, Shivhare RS, Ugale VG (2018) Anti-inflammatory potentials of some novel Murrayanine containing 1,3,4-Oxadiazole derivatives. Asian J Pharm Technol 8(1): 47-51.

15. Mahapatra DK, Chhajed SS, Shivhare RS (2017) Development of Murrayanine-Chalcone hybrids: An effort to combine two privilege scaffolds for enhancing hypoglycemic activity. Int J Pharm Chem Anal 4(2): 30-34.
16. Kanhed AA, Mehere AP, Pandey KR, Mahapatra DK (2016) 4-(2-chloroacetamido) benzoic acid derivatives as local anesthetic agents: design, synthesis, and characterization. UK J Pharm Biosci 4(6): $35-44$.

17. Amdare MD, Jogdand KR, Kathane LL, Kuhite NG, Padole CD, et al. (2017) Synthesis of a potential anti-inflammatory pyrazole derivative from hippuric acid as the starting material. J Pharm Chem Biol Sci 5(3): 216220.

18. Mahapatra DK, Bharti SK (2017) In: Mahapatra DK, Bharti SK (Eds.), Handbook of Research on Medicinal Chemistry. Apple Academic Press, New Jersey, USA.

19. Mahapatra DK, Bharti SK (2016) Drug Design. Tara Publications Private Limited, New Delhi, India.
Creative Commons Attribution 4.0 International License

For possible submissions Click Here

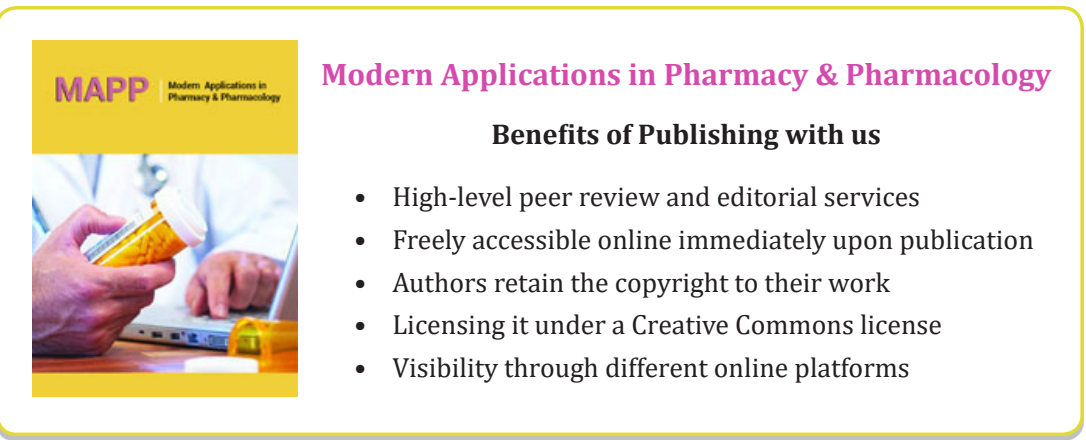

